\title{
Dispersão de Plantas Anuais com Bancos de Sementes: Um Modelo Discreto com Operadores Integrais e Retardamento ${ }^{1}$
}

D.C. MISTRO ${ }^{2}$, L.A.D. RODRIGUES ${ }^{3}$, A.B. SCHMID, Departamento de Matemática, Centro de Ciências Naturais Exatas, Universidade Federal de Santa Maria, 97105-900 Santa Maria, RS, Brasil.

\begin{abstract}
Resumo. Neste trabalho, formulamos um modelo para descrever a dispersão espacial de uma população de plantas anuais com banco de sementes. Consideramos o crescimento da população de plantas dependente da densidade de sementes em germinação, dispersão não local de sementes e banco de sementes de dois anos. O modelo é formulado a partir de equações a diferenças integrais com retardamento. Os efeitos do banco de sementes sobre o comportamento assintótico da solução são analisados através de simulações numéricas. São observadas soluções assintoticamente estáveis, periódicas e caóticas em diagramas de bifurcações construídos numericamente. Neste modelo, o banco de sementes pode ter um efeito estabilizador do equilíbrio da população, ao contrário dos modelos contínuos, em que os retardamentos têm uma influência desestabilizante.
\end{abstract}

\section{Introdução}

Plantas anuais produzem sementes uma vez por ano. A cada ano, as plantas crescem, florescem e morrem, deixando seus descendentes na forma dormente de sementes, que devem sobreviver ao inverno para produzir a nova geração. No ano seguinte uma fração das sementes germina, enquanto outras permanecem dormentes por um ano ou mais antes de germinar.

Edelstein-Keshet [3] formulou um modelo para descrever a dinâmica de plantas anuais considerando um banco de sementes de dois anos. Em seu modelo, considera apenas o número de indivíduos em cada geração, isto é, o meio é considerado espacialmente homogêneo. No entanto, a variabilidade espacial é de grande importância na dinâmica de uma população. O estudo das invasões biológicas é fundamental para se prever os danos ecológicos e econômicos da dispersão de uma população.

Modelos para dispersão de plantas têm sido desenvolvidos através de equações a diferenças integrais $[1,2,4]$. Uma vez que os fenômenos de dispersão e reprodução

\footnotetext{
${ }^{1}$ Projeto financiado pela FAPERGS - Processo 02/1073.2

${ }^{2}$ diomar@ccne.ufsm.br

${ }^{3}$ luizdiaz@ccne.ufsm.br

${ }^{4}$ Bolsista de Iniciação Científica - FAPERGS (Processo 011226)
} 
são eventos discretos para muitas espécies de plantas, este tipo de modelagem é mais adequado do que os modelos de reação-difusão amplamente utilizados para descrever dispersão populacional [9, 10, 11]. Além disso, o operador de difusão pressupõe somente dispersão local de sementes, o que não ocorre para as plantas aqui consideradas.

Os modelos desenvolvidos para dispersão de plantas, em geral, não consideram bancos de sementes. Isto é, consideram que as sementes com mais de um ano não são aptas a germinar ou morrem.

Neste trabalho, a partir dos modelos apresentados por Edelstein-Keshet [3] e Andersen [2] formulamos um modelo discreto com operadores integrais para descrever a dinâmica e dispersão de plantas anuais com bancos de sementes. O modelo considera crescimento dependente da densidade como efeito da competição intraespecífica e o comportamento de dispersão de sementes. São realizados experimentos numéricos, através dos quais concluímos sobre o comportamento assintótico do modelo proposto.

\section{O Modelo}

Consideramos que as plantas produzem sementes no final da estação de crescimento e depois morrem. Uma fração das sementes sobrevive ao inverno e parte delas germina na próxima estação. Das sementes restantes, algumas permanecem em estado latente, para germinar após o segundo inverno. Para simplificar, suporemos inicialmente, que as sementes com mais de dois anos são inviáveis e morrem.

Se $s_{t}$ e $p_{t}$ denotam, respectivamente, o número de sementes em germinação e plantas adultas na geração $t$ em um ambiente espacialmente homogêneo, então

$$
s_{t+1}=\alpha \sigma \gamma p_{t}+\beta \sigma(1-\alpha) \sigma \gamma p_{t-1}
$$

onde $\gamma$ descreve o número de sementes produzidas por planta; $\alpha$ indica a fração de sementes de um ano que germina; $\sigma$ representa a fração de sementes que sobrevive a um inverno e $\beta$, a fração de sementes de dois anos que germina. Assim, o primeiro termo à direita representa as sementes de um ano que germinam e o segundo termo à direita, as sementes de dois anos que sobreviveram e germinam no ano $t+1$. Os parâmetros $\alpha, \beta$ e $\sigma$ são adimensionais enquanto $[\gamma]=\frac{\text { sementes }}{\text { planta }}$.

A cada geração, as sementes em germinação irão competir por alimento, luz e espaço e desta forma a densidade de adultos terá um crescimento dependente da densidade de sementes em germinação. Usando a função de crescimento de Ricker $[3,9]$, a população de plantas na geração $t+1$ é dada por

$$
\begin{aligned}
p_{t+1} & =a s_{t+1} \exp \left(-b s_{t+1}\right) \\
& =a\left(\alpha \sigma \gamma p_{t}+\beta \sigma(1-\alpha) \sigma \gamma p_{t-1}\right) \exp \left(-b\left(\alpha \sigma \gamma p_{t}+\beta \sigma(1-\alpha) \sigma \gamma p_{t-1}\right)\right)
\end{aligned}
$$

onde $a$ indica a fecundidade per capita das sementes em germinação na ausência da dependência da densidade e $b$ representa o grau com que a fecundidade é reduzida pelo efeito da dependência da densidade. Os parâmetros $a$ e $b$ têm as seguintes 
dimensões:

$$
[a]=\frac{\text { planta }}{\text { sementes }}, \quad[b]=\frac{1}{\text { sementes }} .
$$

Agora, para introduzir a variação espacial, vamos considerar $S_{t}(x)$ a densidade de sementes em germinação na posição $x$ e no início da geração $t$ depois que os adultos das duas gerações anteriores propagaram suas sementes e, $P_{t}(x)$ a densidade de plantas adultas na geração $t$ no ponto $x$. Assim, a densidade de sementes é relacionada às plantas adultas pelo operador integral

$$
S_{t+1}(x)=\alpha \sigma \gamma \int_{\Omega} k(x-y) P_{t}(y) d y+\beta \sigma(1-\alpha) \sigma \gamma \int_{\Omega} k(x-y) P_{t-1}(y) d y,
$$

onde $\Omega$ é o domínio unidimensional considerado e $k(x, y)$, chamado núcleo de redistribuição, descreve a probabilidade de uma semente, proveniente das plantas da posição $y$, cair na posição $x$. O núcleo de redistribuição, de fundamental importância neste tipo de formulação, representa os diferentes tipos de comportamento das plantas ao dispersar suas sementes: por lançamento, pelo vento, através de queda simples, etc.

Assumimos aqui que o núcleo de redistribuição depende apenas da distância entre $x$ e $y$ e não explicitamente de sua posição absoluta. Em outras palavras, a dispersão de sementes é homogênea e isotrópica. Assim, no que se segue usaremos $k(x, y)=k(x-y)=k(y-x)$.

Em cada ponto do domínio, as sementes irão competir por espaço, luz e alimento. Dessa forma, para determinar a densidade de plantas adultas na geração $t+1$ na posição $x$, substituímos $S_{t}(x)$ e $P_{t}(x)$ na equação $(2.1)$, obtendo

$$
\begin{aligned}
& P_{t+1}(x)=a S_{t+1}(x) \exp \left(-b S_{t+1}(x)\right) \\
& \quad=a\left(\alpha \sigma \gamma \int_{\Omega} k(x-y) P_{t}(y) d y+\beta \sigma(1-\alpha) \sigma \gamma \int_{\Omega} k(x-y) P_{t-1}(y) d y\right) \\
& \quad \times \exp \left(-b\left(\alpha \sigma \gamma \int_{\Omega} k(x-y) P_{t}(y) d y+\beta \sigma(1-\alpha) \sigma \gamma \int_{\Omega} k(x-y) P_{t-1}(y) d y\right)\right) .
\end{aligned}
$$

Dado o grande número de parâmetros envolvidos no modelo, efetuamos uma adimensionalização, a qual reduz o número de parâmetros a grupos adimensionais reveladores dos reais mecanismos que determinam a dinâmica do problema. Para isto, introduzimos a variável adimensional $U_{t}(x)=\frac{P_{t}(x)}{a / b}$ que, substituída na equação (2.2), fornece a densidade adimensional de sementes na posição $x$ no início da geração $t+1$ :

$$
\bar{S}_{t+1}(x)=A \int_{\Omega} k(x-y) U_{t}(y) d y+A B \int_{\Omega} k(x-y) U_{t-1}(y) d y,
$$

onde

$$
A=a \alpha \sigma \gamma \quad \text { e } \quad B=\frac{\beta \sigma(1-\alpha)}{\alpha}
$$

são constantes adimensionais. 


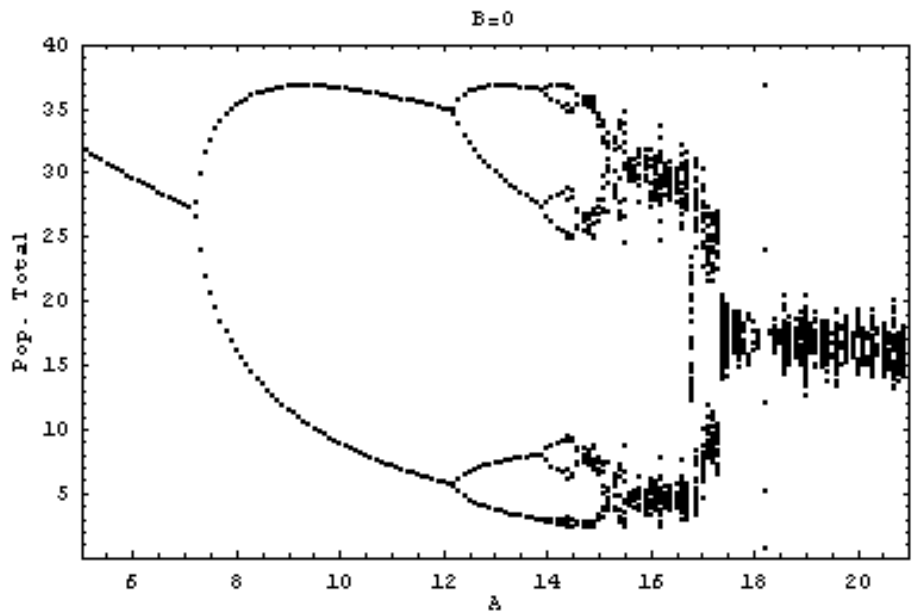

Figura 1: Diagrama de bifurcação com relação a $A$, para $B=0$. O eixo vertical representa a população total após o equilíbrio ter sido alcançado.

Dessa forma,

$$
U_{t+1}(x)=\bar{S}_{t+1}(x) \exp \left(-\bar{S}_{t+1}(x)\right)
$$

$\mathrm{ou}$

$$
\begin{aligned}
& U_{t+1}(x)=A\left(\int_{\Omega} k(x-y) U_{t}(y) d y+B \int_{\Omega} k(x-y) U_{t-1}(y) d y\right) \\
& \quad \times \exp \left(-A\left(\int_{\Omega} k(x-y) U_{t}(y) d y+B \int_{\Omega} k(x-y) U_{t-1}(y) d y\right)\right)
\end{aligned}
$$

é a densidade adimensional de plantas adultas na posição $x$, na geração $t+1$.

$B=0$ na equação (2.6) significa ausência de bancos de sementes, isto é, após um ano as sementes que não germinaram tornam-se inviáveis e morrem. Neste caso, a equação (2.6) torna-se:

$$
U_{t+1}(x)=A \int_{\Omega} k(x-y) U_{t}(y) d y \cdot \exp \left(-A \int_{\Omega} k(x-y) U_{t}(y) d y\right),
$$

análoga adimensional do modelo analisado por Andersen [2]. Para pequenos valores de $A$, em habitats finitos, a solução desta equação converge para um ponto fixo $U(x)$, que representa uma distribuição de equilíbrio homogênea das plantas. À medida que o parâmetro $A$ aumenta, o ponto fixo perde sua estabilidade e ocorre uma bifurcação, surgindo um ciclo de período-2, isto é, a distribuição de equilíbrio das plantas oscila homogeneamente entre dois valores. Aumentos maiores em $A$ causam uma seqüência de bifurcações que conduzem ao caos [2].

O diagrama de bifurcação para a equação (2.7), isto é, para $B=0$ é ilustrado na Figura 1. Neste gráfico, a quantidade representada no eixo vertical é a população total $\bar{U}=\int_{\Omega} U(x) d x$ após o equilíbrio ter sido alcançado. 


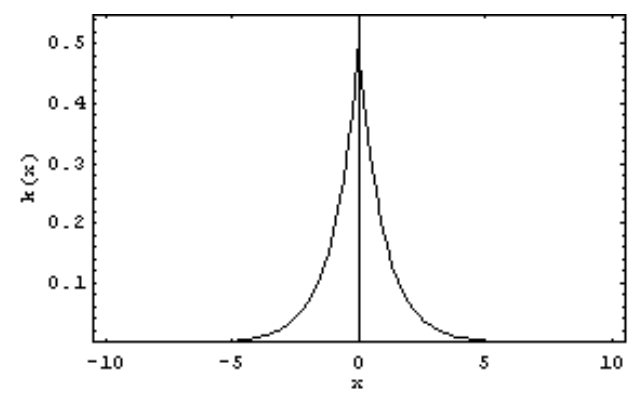

Figura 2: Núcleo de redistribuição de Weibull.

Nosso objetivo, neste trabalho, é estudar o comportamento assintótico do modelo (2.6) para $0 \leq B \leq 1$, ou seja, avaliar os efeitos do banco de sementes na dinâmica da população de plantas adultas. Em termos dos parâmetros dimensionais, temos $0 \leq \frac{\beta \sigma(1-\alpha)}{\alpha} \leq 1$, em outras palavras, a fração de sementes que germina no segundo ano, no máximo, é igual àquela que germinou no primeiro ano, hipótese bastante razoável para um grande número de espécies de plantas anuais [7].

\section{Propriedades do Modelo}

Analisamos o comportamento assintótico da solução da equação (2.6) através do seguinte experimento numérico: consideramos uma distribuição inicial constante $U_{0}(x)=0,25$. A partir desta distribuição inicial, fixamos alguns valores de $A$ escolhidos a partir do comportamento exibido na Figura 1, isto é, valores de $A$ para os quais o comportamento da solução da equação (2.7) é diferente. Para cada $A$ fixo, analisamos a população total de plantas para $0 \leq B \leq 1$, confeccionando, assim, diagramas de bifurcação com relação ao parâmetro $B$. Em todas as simulações utilizamos como núcleo de redistribuição a distribuição de Weibull $k(x)=\frac{1}{2} \exp (-|x|)$ (Figura 2) e um domínio finito de comprimento fixo.

Os resultados ilustrados nos gráficos da Figura 3 mostram que o banco de sementes pode provocar mudanças significativas na dinâmica de plantas anuais. Nestes gráficos, o eixo vertical indica a população total de plantas após o equilíbrio ter sido alcançado, isto é, representamos as últimas cem iterações após mil iterações terem sido executadas.

Para $A=6$ e $B=0$, isto é, na ausência de bancos de sementes, a população assume uma distribuição de equilíbrio homogênea estável. À medida que aumentamos $B$, o valor da população total diminui, porém o equilíbrio continua um ponto fixo estável, como ilustra o gráfico na Figura 3. 

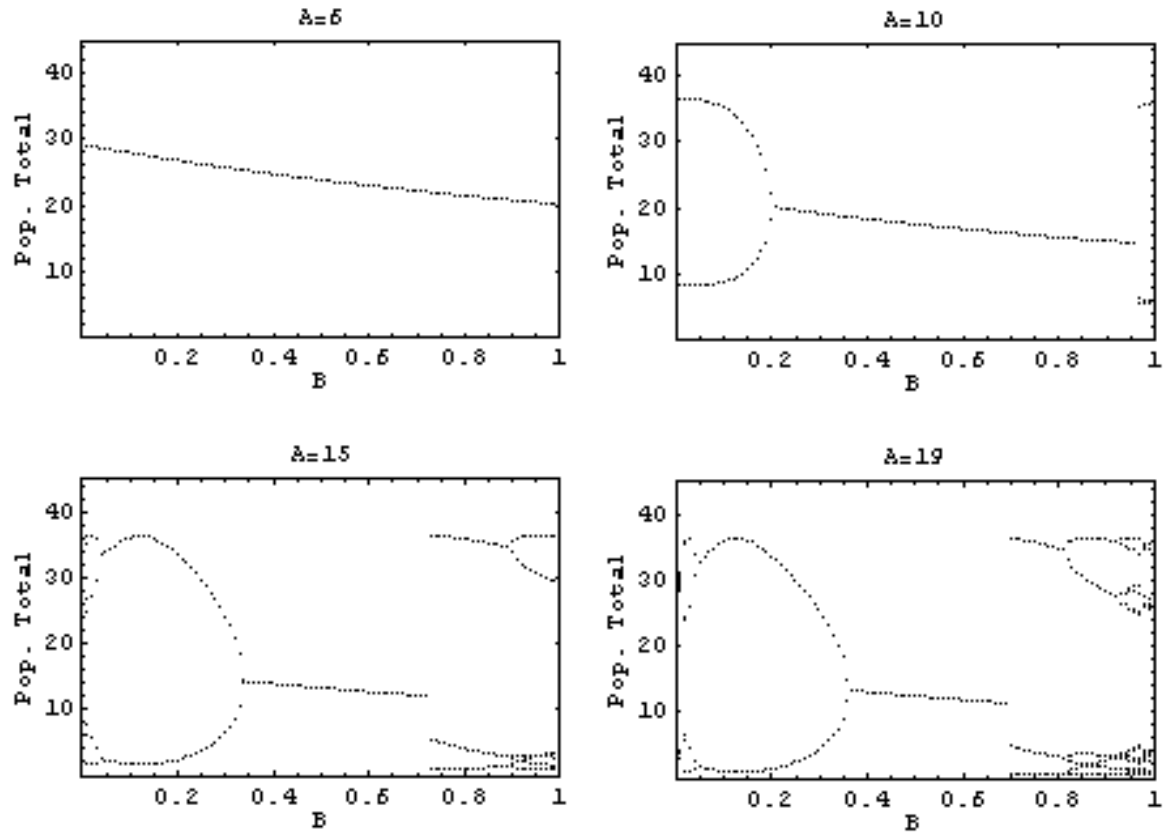

Figura 3: Diagramas de bifurcação com relação a $B$ para diferentes valores de $A$. $\mathrm{O}$ eixo vertical de cada gráfico representa a população total após o equilíbrio ter sido alcançado.

Para $A=10$ e $B=0$, há um ciclo de período dois, isto é, a distribuição espacial de equilíbrio da população oscila homogeneamente entre dois valores. Com o aumento da germinação do banco de sementes, observamos uma mudança qualitativa no comportamento da solução de equilíbrio. Ocorre uma bifurcação em $B=0,21$ e o ciclo de período dois dá lugar a um equilíbrio homogêneo estável até o valor $B=0,98$ ser alcançado quando então, ocorre uma nova bifurcação e surge um ciclo de período três (Figura 3 ).

Com $A=15$ e $B=0$ ocorre um ciclo de período oito. À medida que o parâmetro $B$ é aumentado, surge um ciclo de período quatro, em seguida um ciclo de período dois e para $0,35 \leq B \leq 0,72$ a população total de equilíbrio é estável. Para $0,72 \leq B \leq 0,89$ surge um ciclo de período três e, finalmente, um ciclo de período seis (Figura 3).

Para $A=19$ o comportamento da solução é semelhante. Para aumentos em $B$, surgem ciclos de período cada vez menor e em seguida, um ponto de equilíbrio estável, o qual dá lugar a um ciclo de período três. Novamente ocorrem bifurcações surgindo ciclo de período seis e ciclos de períodos maiores (Figura 3).

No intervalo de $A$ onde a solução de equilíbrio da equação (2.7) tem um ponto fixo estável, a germinação do banco de sementes não altera a estabilidade da solução, apenas diminui a população total de plantas. Nos intervalos de $A$ para os quais o modelo representado pela equação (2.7) assume um regime periódico, aumentos em $B$ provocam uma diminuição na amplitude das oscilações até o surgimento de um 
único ponto de equilíbrio estável. Aumentos maiores em $B$ levam novamente a soluções periódicas.

\section{Conclusões}

Neste trabalho formulamos um modelo discreto com operadores integrais para a descrição da dispersão espacial de plantas com bancos de sementes, o qual considera o crescimento das plantas dependente da densidade de sementes em germinação e o comportamento de espalhamento das plantas. Nas simulações numéricas realizadas, foi observado que a germinação das sementes do banco de sementes pode alterar significativamente a dinâmica da população de plantas adultas.

Os resultados obtidos mostram que, ao contrário dos modelos contínuos em que os retardamentos têm um efeito desestabilizador produzindo soluções periódicas [9], o banco de sementes em modelos discretos integrais, pode ter um efeito estabilizador do equilíbrio da população. Para determinados valores do parâmetro $B$, que representa o efeito da germinação do banco de sementes, soluções periódicas dão lugar a um ponto fixo assintoticamente estável.

Para a condição inicial utilizada, as soluções convergem para distribuições espaciais de equilíbrio homogêneas. Em extensivas simulações numéricas, obtivemos soluções estáveis, ciclos de diferentes períodos e soluções caóticas no tempo sempre com uma distribuição espacial homogênea.

À medida que $A$ (relacionado com as sementes de um ano) aumenta, diminui o intervalo do parâmetro $B$ onde há um equilíbrio estável da população. Isto significa que as populações de espécies que produzem grande quantidade de sementes com baixa mortalidade, alta taxa de germinação no primeiro ano ou alta fecundidade per capita (ou seja, $A=a \alpha \sigma \gamma$ grande), tendem a oscilar se a germinação do banco de sementes não estiver em torno de cinqüenta por cento da germinação do primeiro ano.

Populações de espécies com pequena produtividade de sementes ou alta taxa de mortalidade das sementes (isto é, $A$ pequeno) tendem a atingir um valor de equilíbrio estável, mesmo com alta germinação do banco de sementes.

Observamos também que à medida que $A$ aumenta, as soluções periódicas apresentam amplitudes maiores. No entanto, aumentos em $B$ fazem com que estas oscilações diminuam levando a um único ponto de equilíbrio estável. Aumentos maiores em $B$ novamente levam a soluções oscilantes. Portanto, o banco de sementes não deve ser omitido no estudo da dispersão de plantas anuais.

Em estudos futuros pretendemos analisar o comportamento do modelo (2.6) para outras distribuições iniciais da população de plantas tanto no que se refere à estabilidade da solução de equilíbrio como à distribuição espacial. Resultados iniciais sugerem que, dependendo da distribuição inicial, pode ocorrer a formação de padrões espaciais não homogêneos mesmo na ausência de espécies competidoras ou predadoras que poderiam causar instabilidade difusiva.

Abstract. In this work a spatially explicit integrodifference equation model is studied for the spread of an annual plant population with seed bank. The model 
is capable of predicting stable, cyclical and chaotic asymptotic behavior. We show that the seed bank is very important in determining a species colonizing ability.

\section{Referências}

[1] E.J. Allen, L.J.S. Allen e G. Xiaoning, Dispersal and Competition Models for Plants, Journal of Mathematical Biology, 34 (1996), 455-481.

[2] M. Andersen, Properties of Some Density-Dependent Integrodifference Equation Population Models, Mathematical Biosciences, 104 (1991), 135-157.

[3] L. Edelstein-Keshet, "Mathematical Models in Biology", Random House, New York, 1988.

[4] D.R. Hart e R.H. Gardner, A Spatial Model for the Spread of Invading Organisms Subject to Competition, Journal of Mathematical Biology, 35 (1997), 935-948.

[5] M. Kot e W.M. Schaffer, Discrete-Time Growth-Dispersal Models, Mathematical Biosciences, 80 (1986), 109-136.

[6] M. Kot, Discrete-Time Travelling Waves: Ecological Exemples, Journal of Mathematical Biology, 30 (1992), 413-430.

[7] N. MacDonald e A.R. Watkinson, Models of an Annual Plant Population with a Seed Bank, Journal of Theoretical Biology, 93 (1981), 643-653.

[8] D.C. Mistro, "Modelos para Dispersão de Abelhas Africanizadas: Um Zoom Matemático", Tese de Doutorado, Matemática Aplicada, IMECC, UNICAMP, 1998.

[9] J.D. Murray, "Mathematical Biology", Springer-Verlag, Berlin, 1989.

[10] A. Okubo, "Diffusion and Ecological Problems: Mathematical Models", Springer-Verlag, Berlin, 1980.

[11] N. Shigesada e K. Kawasaki, "Biology Invasions: Theory and Practice", Oxford University Press, 1997. 